\title{
Oral Contraceptives and the Small Increased Risk of Breast Cancer
}

\author{
David J. Hunter, M.B., B.S., Sc.D.
}

In this issue of the Journal, Morch et al. ${ }^{1}$ present the results of a large prospective study of the association between the use of hormonal contraceptives and the risk of breast cancer among women in Denmark who were younger than 50 years of age. They observed a $20 \%$ higher risk among women who were currently using or had recently used hormonal contraceptives than among those who had never used them, and the risk increased with a longer duration of use.

The association between the current use of oral contraceptives and breast cancer is well established; the relative risk of 1.20 in the current analysis is similar to that reported by the Collaborative Group on Hormonal Factors in Breast Cancer $^{2}$ and in previous prospective studies such as the Nurses' Health Studies. ${ }^{3,4}$ The advantage of the analysis by Mprch et al. is that most of the formulations used were those that have been prevalent in Denmark since 1995; the Collaborative Group data were based on the use of formulations in the 1980s and earlier. The study by Morch and colleagues confirms that the increased breast-cancer risk of approximately 20\% among women who were currently using oral contraceptives, a risk that was initially reported with the use of older, often higher-dose formulations, also applies to contemporary formulations of oral contraceptives.

In an apparent contrast, the results of a Centers for Disease Control and Prevention (CDC) case-control study reported by Marchbanks et al. in the Journal in 2002 showed no significant increase in the risk of breast cancer ${ }^{5}$; however, owing to the age range of the women involved in that study (35 to 64 years), almost all use of oral contraceptives was among women who had used them in the past, and the upper bound of the $95 \%$ confidence interval for current use of 1.3 in the CDC study comfortably includes the relative risk of 1.20 in the present study. In 2007, the International Agency for Research on Cancer concluded that there was sufficient evidence to establish the carcinogenicity of combined oral estrogen-progestin contraceptives in humans, with an increased risk of breast cancer limited to women who were currently using or had recently used them. ${ }^{6}$ In the current study, there was a suggestion that risk may persist more than 5 years after discontinuation of hormonal contraception among women who had used hormonal contraceptives for at least 5 years, but this should be regarded as preliminary; the increase in risk would not have been significant with adjustment for multiple comparisons involving different categories of duration and time since last use.

Thus, the main result of the study is expected. The much larger sample size than in previous studies permits the examination of subgroups for which previous prospective assessments have been limited. A clear duration-response association was observed, supporting the credibility of the findings. The association was also significant among women younger than 35 years of age and among nulliparous women.

The most important subgroup analyses in the current study involved the risks associated with the various formulations used, particularly various progestins. Although there are some differences in the relative risks, all the confidence intervals overlap the consensus estimate of 1.20. Thus, these results do not suggest that any particular preparation is free of risk. Notably, the associations between the levonorgestrel-only oral formulation and the levonorgestrel-releasing intrauterine device (IUD) and breast-cancer risk were unequivocally positive.

What, then, are the implications? First, the approximately $20 \%$ higher risk of breast cancer among women who currently use hormonal contraceptives and those who do not must be placed in the context of the low incidence rates of breast cancer among younger women. As the authors show, owing to the risk of breast cancer that is more than 5 times as high among women in their 40 s as among women in their 30 s, the excess number of cases of breast cancer associated with the use of hormonal contraceptives increases rapidly with age. The absolute increase in risk is 
13 per 100,000 women overall, but only 2 per 100,000 women younger than 35 years of age; most of the cases that occurred in this analysis occurred among women who were using oral contraceptives in their 40s.

Second, the risk of breast cancer needs to be balanced against the benefits of the use of oral contraceptives. Beyond the fact that they provide an effective means of contraception and may benefit women with dysmenorrhea or menorrhagia, the use of oral contraceptives is associated with substantial reductions in the risks of ovarian, endometrial, and colorectal cancers later in life. Indeed, some calculations have suggested that the net effect of the use of oral contraceptives for 5 years or longer is a slight reduction in the total risk of cancer. ${ }^{7}$ The higher excess risk as women move into their 40s - of breast cancer as well as of other uncommon risks such as myocardial infarction and stroke - suggests careful consideration of alternative methods of contraception such as nonhormonal, long-acting, reversible contraceptives (e.g., IUDs) in this age group.

Third, these data suggest that the search for an oral contraceptive that does not elevate the risk of breast cancer needs to continue. In the 1980s and 1990s, there was some optimism regarding the development of a formulation that would reduce a woman's risk of breast cancer, ${ }^{8}$ but research into this possibility appears to have stalled.

Finally, this study exemplifies the opportuni- ties to use population-wide "big data" approaches to evaluate very important issues at a low cost. Investigators in countries that have universal access to health care and those who have community agreement for the linkage of various databases are better able to conduct research that clarifies both the risks and benefits of common exposures, including prescription medicines.

Disclosure forms provided by the author are available with the full text of this editorial at NEJM.org.

From the Nuffield Department of Population Health, University of Oxford, Oxford, United Kingdom.

1. Mørch LS, Skovlund CW, Hannaford PC, Iversen L, Fielding S, Lidegaard $\Phi$. Contemporary hormonal contraception and the risk of breast cancer. N Engl J Med 2017;377:2228-39.

2. Collaborative Group on Hormonal Factors in Breast Cancer. Breast cancer and hormonal contraceptives: further results. Contraception 1996;54:Suppl:1S-106S.

3. Romieu I, Willett WC, Colditz GA, et al. Prospective study of oral contraceptive use and risk of breast cancer in women. J Natl Cancer Inst 1989;81:1313-21.

4. Hunter DJ, Colditz GA, Hankinson SE, et al. Oral contraceptive use and breast cancer: a prospective study of young women. Cancer Epidemiol Biomarkers Prev 2010;19:2496-502.

5. Marchbanks PA, McDonald JA, Wilson HG, et al. Oral contraceptives and the risk of breast cancer. N Engl J Med 2002;346: 2025-32.

6. IARC Working Group on the Evaluation of Carcinogenic Risks to Humans. Combined estrogen-progestogen contraceptives and combined estrogen-progestogen menopausal therapy. IARC Monogr Eval Carcinog Risks Hum 2007;91:1-528.

7. Bassuk SS, Manson JE. Oral contraceptives and menopausal hormone therapy: relative and attributable risks of cardiovascular disease, cancer, and other health outcomes. Ann Epidemiol 2015;25:193-200.

8. Pike MC, Spicer DV. Hormonal contraception and chemoprevention of female cancers. Endocr Relat Cancer 2000;7:73-83. DOI: 10.1056/NEJMe1709636

Copyright () 2017 Massachusetts Medical Society. 